\title{
In vivo assessment of bifurcation optimal viewing angles and bifurcation angles by three-dimensional (3D) quantitative coronary angiography
}

\author{
Shengxian Tu $\cdot$ Jing Jing $\cdot$ Niels R. Holm $\cdot$ Kevin Onsea $\cdot$ Tao Zhang $\cdot$ \\ Tom Adriaenssens $\cdot$ Christophe Dubois $\cdot$ Walter Desmet $\cdot$ Leif Thuesen • \\ Yundai Chen · Johan H. C. Reiber
}

Received: 24 August 2011/Accepted: 2 December 2011

(C) The Author(s) 2011. This article is published with open access at Springerlink.com

\begin{abstract}
Evaluation and stenting of coronary bifurcation lesions may benefit from optimal angiographic views. The anatomy-defined bifurcation optimal viewing angle (ABOVA) is characterized by having an orthogonal view of the bifurcation, such that overlap and foreshortening at the ostium are minimized. However, due to the mechanical constraints of the X-ray systems, certain deep angles cannot be reached by the $\mathrm{C}$-arm. Therefore, second best or, socalled obtainable bifurcation optimal viewing angle (OBOVA) has to be used as an alternative. This study
\end{abstract}

Shengxian Tu and Jing Jing equally contributed to the writing of the manuscript.

S. Tu · J. H. C. Reiber

Division of Image Processing, Department of Radiology, Leiden University Medical Center, Albinusdreef 2, 2300 RC Leiden, The Netherlands e-mail: S.T.Tu@lumc.nl

J. Jing · T. Zhang · Y. Chen $(\bowtie)$

Department of Cardiology, Chinese PLA General

Hospital, No. 28 Fuxin Road, Haidian District, Beijing

100853, China

e-mail: cyundai@medmail.com.cn

N. R. Holm · L. Thuesen

Department of Cardiology, Aarhus University Hospital, Skejby, Denmark

K. Onsea - T. Adriaenssens - C. Dubois · W. Desmet Department of Cardiovascular Diseases, University

Hospitals Leuven, Leuven, Belgium assessed the distributions of ABOVA and OBOVA using $3 \mathrm{D}$ quantitative coronary angiography in a typical patient population. In addition, the bifurcation angles in four main coronary bifurcations were assessed and compared. Patients with obstructive coronary bifurcation disease were included in this multicenter registry. A novel and validated 3D QCA software package was applied to reconstruct the bifurcations and to calculate the bifurcation angles in 3D. A list of optimal viewing angle candidates including ABOVA was also automatically proposed by the software. In a next step, the operator selected the best viewing angle as OBOVA, while applying a novel overlap prediction approach to assure no overlap between the target bifurcation and other major coronary arteries. A total of 194 bifurcations from 181 patients were assessed. The ABOVA could not be reached in $56.7 \%$ of the cases; being $40(81.6 \%), 40$ (78.4\%), 9 (17.6\%), and $21(48.8 \%)$ cases for LM/ LAD/LCx, LAD/Diagonal, LCx/OM, and PDA/PLA, respectively. Both ABOVA and OBOVA distributed sparsely with large ranges of variance: $\mathrm{LM} / \mathrm{LAD} / \mathrm{LCx}$, $5 \pm 33$ RAO, $47 \pm 35$ Caudal versus $4 \pm 39$ LAO, $35 \pm 16$ Caudal; LAD/Diagonal, $4 \pm 38$ RAO, $50 \pm 14$ Cranial versus $14 \pm 28 \mathrm{LAO}, 33 \pm 5$ Cranial; LCx/OM, $21 \pm 32 \mathrm{LAO}, 27 \pm 17$ Caudal versus $18 \pm 31$ LAO, $25 \pm 13$ Caudal; PDA/PLA, $34 \pm 21$ LAO, $36 \pm 21$ Cranial versus $28 \pm 25$ LAO, $29 \pm 15$ Cranial. LM/LAD/LCx had the smallest proximal bifurcation angle $\left(128^{\circ} \pm 24^{\circ}\right)$ and the largest distal bifurcation angle $\left(80^{\circ} \pm 21^{\circ}\right)$, as compared with 
LAD/Diagonal $\left(151^{\circ} \pm 13^{\circ}\right.$ and $\left.48^{\circ} \pm 16^{\circ}\right), \mathrm{LCx} /$ $\mathrm{OM}\left(146^{\circ} \pm 18^{\circ}\right.$ and $\left.57^{\circ} \pm 16^{\circ}\right)$, and PDA/PLA $\left(145^{\circ} \pm 19^{\circ}\right.$ and $\left.59^{\circ} \pm 17^{\circ}\right)$. In conclusion, large variabilities in optimal viewing angles existed for all main coronary bifurcations. The anatomy-defined bifurcation optimal viewing angle could not be reached in vivo in roughly half of the cases due to the mechanical constraints of the current X-ray systems. Obtainable bifurcation optimal viewing angle should be provided as an alternative or second best. The bifurcation angles in the left main bifurcation demonstrated the largest variabilities.

Keywords Coronary artery disease - Quantitative coronary angiography $\cdot$ Three-dimensional reconstruction $\cdot \mathrm{X}$-ray angiography

$\begin{array}{ll}\text { Abbreviations } \\ \text { ABOVA } & \begin{array}{l}\text { Anatomy-defined bifurcation optimal } \\ \text { viewing angle }\end{array} \\ \text { DBA } & \begin{array}{l}\text { Distal bifurcation angle } \\ \text { Digital imaging and communications in } \\ \text { DICOM }\end{array} \\ & \begin{array}{l}\text { medicine } \\ \text { ED }\end{array} \\ \text { EVA } & \text { Expert viewing angle } \\ \text { LAD } & \text { Left anterior descending } \\ \text { LAO } & \text { Left anterior oblique } \\ \text { LCx } & \text { Left circumflex artery } \\ \text { LM } & \text { Left main } \\ \text { OBOVA } & \text { Obtainable bifurcation optimal viewing } \\ & \text { angle } \\ \text { OM } & \text { Obtuse marginal } \\ \text { PBA } & \text { Proximal bifurcation angle } \\ \text { PCI } & \text { Percutaneous coronary interventions } \\ \text { PDA } & \text { Posterior descending artery } \\ \text { PLA } & \text { Posterolateral artery } \\ \text { QCA } & \text { Quantitative coronary angiography } \\ \text { RAO } & \text { Right anterior oblique } \\ \text { RCA } & \text { Right coronary artery } \\ \text { SVA } & \text { Software viewing angle }\end{array}$

\section{Introduction}

Bifurcation lesions are frequent and account for approximately $15-20 \%$ of all percutaneous coronary interventions (PCI) performed worldwide $[1,2]$. The treatment remains challenging with a tendency towards increased restenosis and stent thrombosis [3]. Correct assessment of bifurcation lesion anatomy, especially the ostia of branches, is essential in the choice of treatment strategy. Indeed, meticulous positioning of a sidebranch stent is of uttermost importance to ensure complete ostial lesion coverage and to limit the protrusion of the stent in the main vessel. Currently, X-ray coronary angiography is predominantly used in daily routine to establish the diagnosis and guide PCI. The conventional approach for diagnostic angiography uses a rigid set of multiple standard angiographic views [4], while the modern approach requires immediate interpretation of the first angiographic images, followed by the acquisition of those views that maximally expose the lesion severity and preferably with minimal overlap and foreshortening. In order to obtain the optimal views, operators will interactively adjust the rotation angle (left anterior oblique/right anterior oblique, LAO/RAO) and the angulation angle (Cranial/Caudal) guided by the X-ray images. This "trial-and-error" approach could significantly increase the volume of contrast medium used and the radiation exposure to the patient and staff. Besides, due to the variable anatomy of each individual patient combined with the variable orientation of the heart in the thorax, the chosen angle can be quite different from the true optimal viewing angle $[5,6]$.

Recently developed three-dimensional quantitative coronary angiography (3D QCA) systems enabled the automated determination of bifurcation optimal viewing angles, i.e., the angle characterized by having an orthogonal view of the bifurcation including the ostium, minimizing the vessel foreshortening and overlap [7]. However, this orthogonal view is uniquely determined by the anatomy of the individual bifurcation. Due to the mechanical constraints of the X-ray systems, this anatomy-defined bifurcation optimal viewing angle (ABOVA) might have deep angles, which possibly cannot be reached by the $\mathrm{C}$-arm or might be associated with an unacceptable radiation exposure to the operator. In addition, the possible overlap by other major coronary arteries could significantly influence the visualization of the bifurcation, rendering such an ABOVA less useful [5]. Therefore, second best or, obtainable bifurcation optimal viewing angle (OBOVA) has to be used as an alternative to resolve the aforementioned limitations. This study assessed the distributions of ABOVA and OBOVA using three-dimensional quantitative coronary 
angiography (3D QCA) in four main coronary bifurcations. The proximal bifurcation angle, i.e., the takeoff angle, and the distal bifurcation angle, i.e., the carina angle, at the end-diastolic (ED) phase in these four main coronary bifurcations were also assessed and compared.

\section{Methods}

Study population

A total of 187 patients with obstructive coronary bifurcation disease in four main coronary bifurcations (LM/LAD/LCx, LAD/Diagonal, LCx/OM, and PDA/ PLA) were retrospectively included in this study at three medical centers (Department of Cardiology, Chinese PLA General Hospital, Beijing, China; Department of Cardiology, Aarhus University Hospital, Skejby, Aarhus, Denmark; and Department of Cardiovascular Diseases, University Hospitals Leuven, Leuven, Belgium). Inclusion criteria were: (1) $\mathrm{X}$-ray angiographic images were acquired by digital image intensifiers (flat-panel systems); (2) Two angiographic projections at least $25^{\circ}$ apart with the lumen well filled with contrast dye were recorded before the target bifurcation was revascularized; and (3) The bifurcation was not totally occluded.

Angiographic images were recorded by different $\mathrm{X}$-ray systems (AXIOM-Artis, siemens medical systems, Erlangen, Germany; AlluraXper, philips medical systems, Best, The Netherlands; and Innova 3100, GE Medical Systems, USA). X-ray images were stored in DICOM format at a resolution of $512 \times 512$ or $1,024 \times 1,024$ pixels. All parameters required by the $3 \mathrm{D}$ angiographic reconstruction were automatically recorded by the X-ray systems.

\section{Bifurcation optimal viewing angles}

An optimal viewing angle in the X-ray angiographic systems consists of two parts: rotation angle (LAO/ RAO) and angulation angle (Cranial/Caudal). In this study, the viewing angle was defined as not reachable by the C-arm if the rotation angle was larger than 90 $\mathrm{LAO}$ or $50 \mathrm{RAO}$, or if the angulation angle was larger than 40 Cranial or 40 Caudal.

Three-dimensional angiographic reconstruction was performed using a novel and validated 3D QCA software package (prototype version, Medis medical imaging systems bv, Leiden, The Netherlands) [8, 9]. The 3D bifurcation reconstruction procedure consisted of the following steps: (1) two image sequences acquired at two arbitrary angiographic views with projection angles at least $25^{\circ}$ apart were loaded; (2) properly contrast-filled ED frames of these angiographic image sequences were selected; (3) one to three anatomical markers, e.g., bifurcations, were identified as reference points in the two angiographic views for the automated correction of angiographic system distortions [7]; (4) the target bifurcation was defined and automated 2D lumen edge detection was performed using our extensively validated QCA algorithms [10, 11]; and (5) automated 3D reconstruction and modeling techniques were performed. The resulting bifurcation surface modeled with bean-shape cross-sections in the bifurcation core and elliptical cross-sections in the three segments was generated and visualized in a color-coded fashion. Bifurcation angles and a list of optimal viewing angle candidates including the ABOVA were automatically reported. The ABOVA was characterized by having an orthogonal view of the bifurcation [7], and in such a way that the foreshortening and overlap between the main vessel and the sidebranch at the ostium were minimized. However, overlaps by other major coronary arteries could still deteriorate the quality of the projection when using ABOVA as the projection angle. Therefore, in a next step, a novel overlap prediction algorithm described in our previous study [5] was used to predict the overlap condition.

An example of correcting system distortions in the image geometry for the $3 \mathrm{D}$ angiographic reconstruction is given in Fig. 1. The two bifurcations in the left anterior descending artery (LAD) were identified as reference points and their epipolar lines, being the projection of the X-ray beam directed towards a particular point on one of the projections onto the second projection [12], were superimposed in the two angiographic views by Fig. 1a, b. Due to the system distortions, the epipolar lines did not go through their corresponding reference points. Figure 1a', b' show the results after the automated correction of system distortions: The epipolar lines now go right through their corresponding reference points in both angiographic views, demonstrating the success of this automated procedure. 

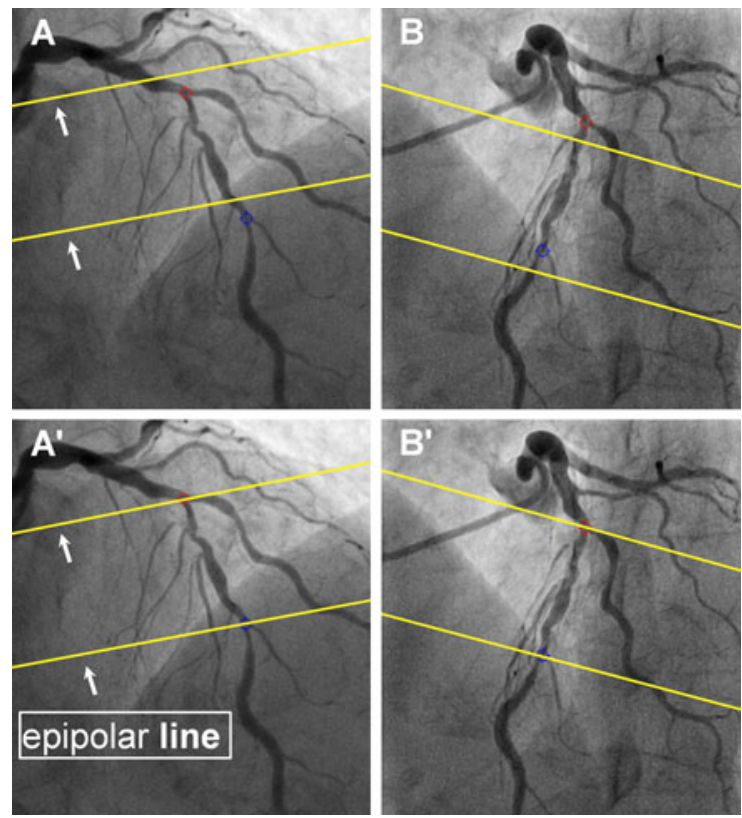

Fig. 1 Automated correction of system distortions in the image geometry for the $3 \mathrm{D}$ angiographic reconstruction: $\mathbf{a}$, b were the two angiographic views (15 RAO, 33 Cranial and 31 LAO, 31 Cranial) used for the $3 \mathrm{D}$ reconstruction. The two epipolar lines did not go through their corresponding reference points, being the red and blue landmarks at the bifurcations, indicating system distortions were present. a', b' show the results after the automated correction of the system distortions: The two epipolar lines now go right through their corresponding red and blue reference points in both angiographic views

Figure 2 shows the two angiographic views with the overlap prediction results and the reconstructed LAD/Diagonal bifurcation in 3D. Figure 2a, b show the two angiographic views (15 RAO, 33 Cranial and 31 LAO, 31 Cranial) with lumen contours superimposed on the $\mathrm{LAD} /$ Diagonal bifurcation and the result of the overlap prediction at ABOVA, being $7 \mathrm{RAO}, 55$ Cranial. The two trajectories (blue lines) in A and $\mathrm{B}$ did not intersect with the same region of any major coronary artery in the two angiographic views, indicating that there was no overlap between the obstructed subsegment of the LAD and other major coronary arteries at ABOVA. $\mathrm{C}$ shows the reconstructed LAD/Diagonal bifurcation at ABOVA. The proximal and distal bifurcation angles were $161^{\circ}$ and $57^{\circ}$, respectively. The subsegment of the LAD between the two green markers had a length of $14.9 \mathrm{~mm}$ in 3D and a foreshortening of $4.9 \%$ at ABOVA. The overlap between the LAD and the Diagonal at the ostium was minimal. However, despite the fact that this ABOVA is characterized by minimal foreshortening and overlap, this ABOVA cannot be reached by the $\mathrm{C}$-arm for practical, mechanical reasons. Therefore, a second best or, another obtainable viewing angle with limited foreshortening and minimal overlap was selected from the list of optimal viewing angle candidates as OBOVA. In this case, 9 LAO, 40 Cranial was chosen as OBOVA and the LAD/Diagonal bifurcation at OBOVA is shown in C. Although the subsegment of the LAD had more foreshortening at OBOVA as compared with ABOVA (11.4 vs. $4.9 \%$ ), the overlap between the LAD and the diagonal at the ostium was still minimal. Figure 2a', b' show the overlap prediction result for the subsegment with other unreconstructed coronary arteries. The shifting centerlines (red curves) along the trajectories (blue lines) in the two angiographic views did not intersect with any major coronary artery at the same time, indicating that there was no overlap between the subsegment of the LAD and other major coronary arteries at OBOVA. In other words, OBOVA was associated with minimal overlap and slightly more foreshortening as compared with ABOVA. However, a very important practical issue is that it can be reached by the X-ray systems.

The 3D angiographic reconstruction and analyses were performed independently by four well-trained 3D QCA analysts. To guarantee the reliability of the 3D bifurcation reconstruction, all reconstruction results were reviewed by one experienced 3D QCA analyst. If the results were considered unreliable, the cases were excluded. Unreliability was mainly caused by: (1) The anatomical markers, e.g., bifurcations, used to correct system distortions were not accurately identified in the two angiographic images due to vessel overlap; (2) Suboptimal correspondence between the two angiographic views was established when the socalled perspective viewing angle, i.e., the angle between the epipolar line and the long-axis of the vessel [8], was almost zero for the entire vessel segment.

\section{Statistics}

Quantitative data were presented as mean difference \pm standard deviation. Due to the unique anatomy of the left main bifurcation, the comparison of bifurcation angles among the four main coronary bifurcations (groups) was performed by the following 

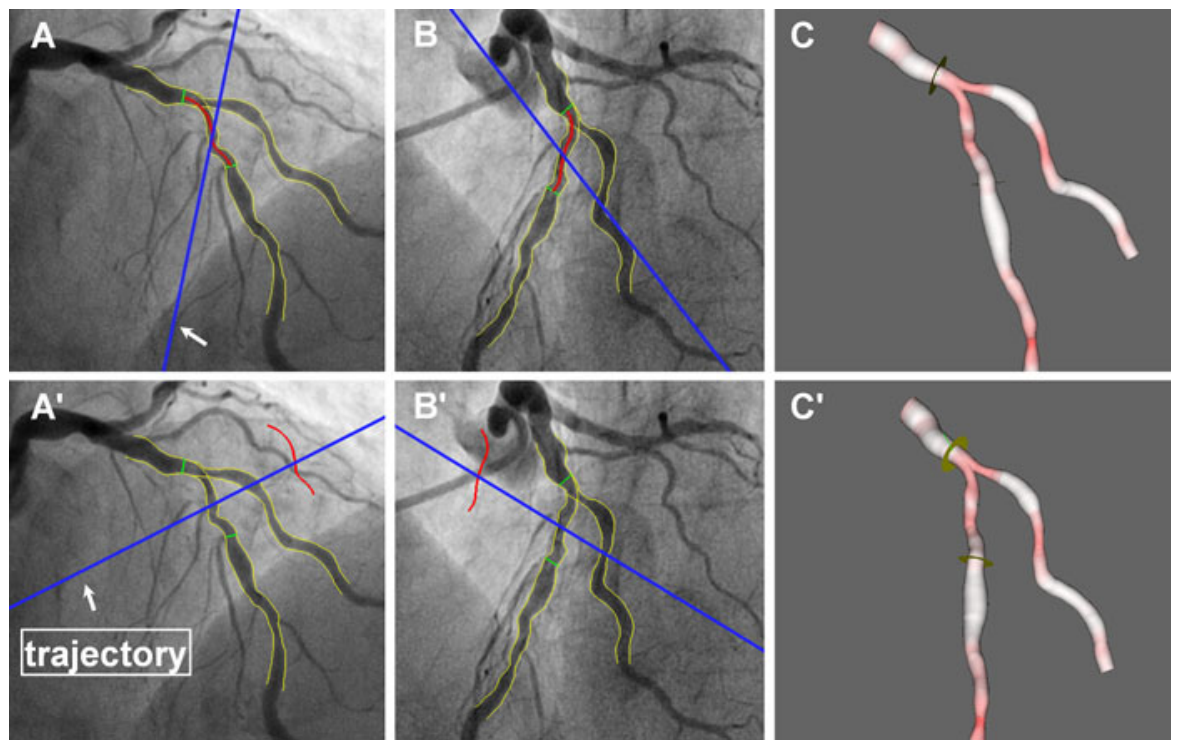

Fig. 23 D reconstructed bifurcation at anatomically defined bifurcation optimal viewing angle (ABOVA) and obtainable bifurcation optimal viewing angle (OBOVA): a, b shows the two angiographic views with lumen contours superimposed on the LAD/Diagonal bifurcation and the overlap prediction result at ABOVA, being $7 \mathrm{RAO}, 55$ Cranial. The trajectories (blue lines) indicated that there was no overlap between the obstructed subsegment of LAD and other major coronary arteries at ABOVA. $\mathbf{c}$ shows the reconstructed bifurcation at ABOVA. The subsegment of LAD between the two green markers had a length

procedure: The left main bifurcation group was compared with each of the other three groups using Mann-Whitney U test. In addition, Kruskal-Wallis U test was used initially to test the overall equality of means in the other three groups. Multiple pairwise comparisons of group means were then carried out using Mann-Whitney $U$ test. A 2 -sided $P$-value of $<0.05$ was considered to be significant. All statistical analyses were carried out using SPSS software package (PASW version 18.0.0, 2009; SPSS Inc, Chicago, IL, USA.).

\section{Results}

A total of 200 bifurcations with obstructive coronary disease were included for analysis. Of all, the analyses of six bifurcations were excluded since the reliability of the 3D bifurcation reconstruction was not approved by the experienced 3D QCA analyst, resulting in a total of 194 bifurcations from 181 patients in the final analyses. Baseline characteristics are given in Table 1. of $14.9 \mathrm{~mm}$ in 3D and a foreshortening of $4.9 \%$ at ABOVA. a' and b' shows the overlap prediction result at OBOVA, being 9 LAO, 40 Cranial. The trajectories (blue lines) and the shifting centerlines (red curves) along the trajectories indicated that there was no overlap between the subsegment and other major coronary arteries at OBOVA. c' shows the reconstructed bifurcation at OBOVA. The same subsegment of LAD had a foreshortening of $11.4 \%$. There was no overlap between the LAD and the Diagonal at the ostium

Table 1 Baseline characteristics

\begin{tabular}{ll}
\hline Patient & $\mathrm{n}=181$ \\
Age & $61(39-88)$ \\
Male/female & $145 / 36$ \\
Bifurcation & $\mathrm{n}=194$ \\
LM/LAD/LCx & $49(25.3 \%)$ \\
LAD/Diagonal & $51(26.3 \%)$ \\
LCx/OM & $51(26.3 \%)$ \\
PDA/PLA & $43(22.2 \%)$ \\
Lesion classifications ${ }^{\mathrm{a}}$ & \\
$(1,0,0)$ & $14(7.2 \%)$ \\
$(0,1,0)$ & $35(18.0 \%)$ \\
$(0,0,1)$ & $14(7.2 \%)$ \\
$(1,1,0)$ & $33(17.0 \%)$ \\
$(1,0,1)$ & $19(9.8 \%)$ \\
$(0,1,1)$ & $23(11.9 \%)$ \\
$(1,1,1)$ & $56(28.9 \%)$
\end{tabular}

$L M$ left main, $L A D$ left anterior descending, $L C x$ left circumflex artery, $O M$ obtuse marginal, $R C A$ right coronary artery, $P D A$ posterior descending artery, $P L A$ posterolateral artery

${ }^{a}$ Medina classification by visual assessment 
In short, the assessed bifurcations included 49 (25.3\%) LM/LAD/LCx, $51 \quad(26.3 \%) \quad$ LAD/Diagonal, 51 (26.3\%) LCx/OM, 43 (22.2\%) PDA/PLA. The ostium of the daughter branches was involved in $147(75.8 \%)$ (main vessel) and 112 (57.7\%) (sidebranch).

The overlap of the bifurcation with other major coronary arteries at ABOVA was uncommon. Only 3 PDA/PLA bifurcations had overlap with the proximal right coronary artery (RCA), while the other three main coronary bifurcations had no overlap when projected at ABOVA. However, ABOVA could not be reached in $110(56.7 \%)$ of the cases; being $40(81.6 \%)$, 40 (78.4\%), 9 (17.6\%), and 21 (48.8\%) cases for LM/ LAD/LCx, LAD/Diagonal, LCx/OM, and PDA/PLA, respectively. Figures 3 and 4 show the distributions of ABOVA and OBOVA in the four main coronary bifurcations, respectively. Quantitative data are presented in Table 2. Both ABOVA and OBOVA distributed sparsely with large ranges of variation for all the main coronary bifurcations: LM/LAD/LCx, $5 \pm 33 \mathrm{RAO}, 47 \pm 35$ Caudal versus $4 \pm 39 \mathrm{LAO}$, $35 \pm 16$ Caudal; LAD/Diagonal, $4 \pm 38$ RAO, $50 \pm 14$ Cranial versus $14 \pm 28$ LAO, $33 \pm 5$ Cranial; $\mathrm{LCx} / \mathrm{OM}, 21 \pm 32 \mathrm{LAO}, 27 \pm 17$ Caudal versus $18 \pm 31$ LAO, $25 \pm 13$ Caudal; PDA/PLA, $34 \pm 21$ LAO, $36 \pm 21$ Cranial vs $28 \pm 25 \mathrm{LAO}, 29 \pm 15$ Cranial.

The bifurcation angles could not be calculated in four cases of the left main bifurcations due to a very short

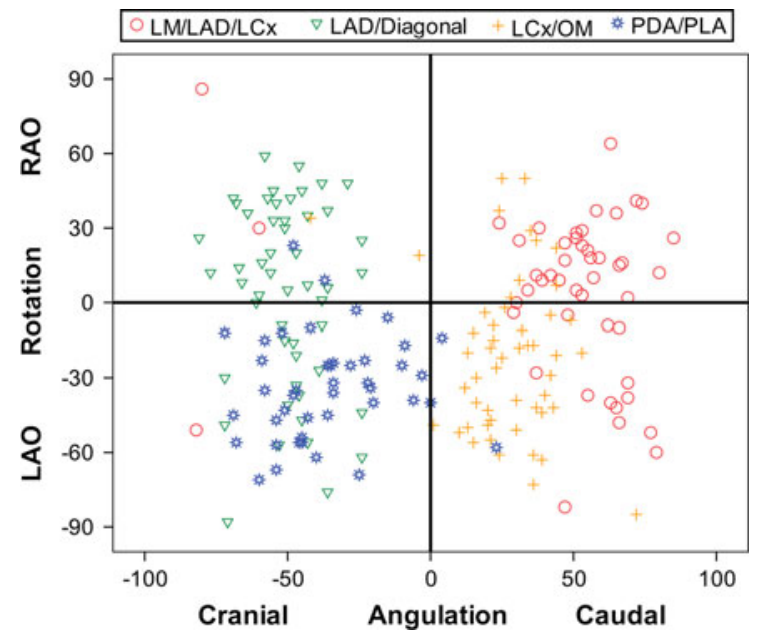

Fig. 3 The distribution of the anatomy-defined bifurcation optimal viewing angle (ABOVA): The ABOVA distributed sparsely with large ranges of variation for all main coronary bifurcations. $n=194$

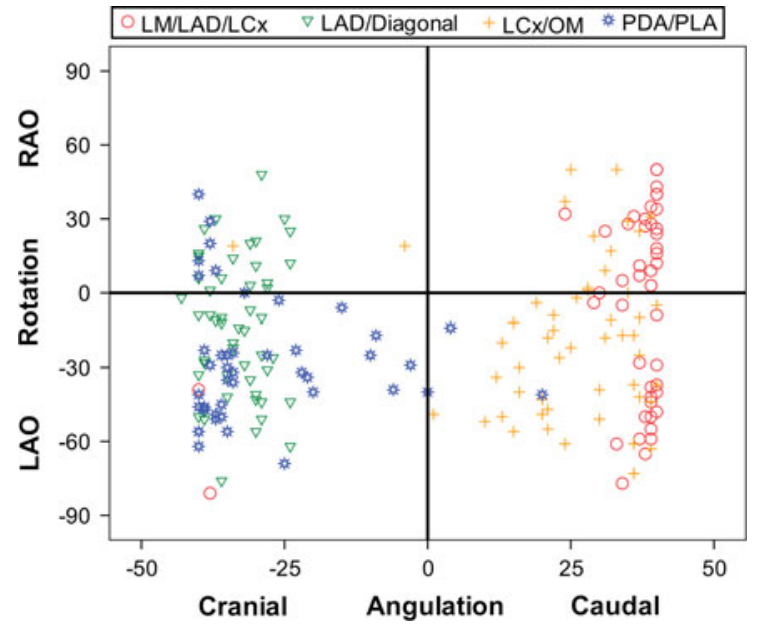

Fig. 4 The distribution of the obtainable bifurcation optimal viewing angle (OBOVA): The OBOVA distributed sparsely with large ranges of variation for all main coronary bifurcations. $n=194$

left main trunk. In the remaining 190 bifurcations, the proximal bifurcation angle (PBA) in LM/LAD/LCx was smaller than any of the other three bifurcations, being $128^{\circ} \pm 24^{\circ}$ versus $151^{\circ} \pm 13^{\circ}(P<0.001)$ in $\mathrm{LAD} /$ Diagonal, $146^{\circ} \pm 18^{\circ}(P<0.001)$ in $\mathrm{LCx} / \mathrm{OM}$, and $145^{\circ} \pm 19^{\circ}(P=0.001)$ in PDA/PLA, respectively. The distal bifurcation angle (DBA) in LM/LAD/LCx was larger than any of the other three bifurcations, being $80^{\circ} \pm 21^{\circ}$ versus $48^{\circ} \pm 16^{\circ}(P<0.001)$ in LAD/diagonal, $57^{\circ} \pm 16^{\circ}(P<0.001)$ in $\mathrm{LCx} / \mathrm{OM}$, and $59^{\circ} \pm$ $17^{\circ}(P<0.001)$ in PDA/PLA, respectively. The PBAs in $\mathrm{LAD} /$ diagonal, $\mathrm{LCx} / \mathrm{OM}$, and PDA/PLA were not statistically different $(P=0.133)$. However, the DBA in LAD/Diagonal was smaller as compared with LCx/ $\mathrm{OM}(P=0.004)$ and PDA/PLA $(P=0.001)$, while the DBAs in LCX/OM and PDA/PLA were not statistically different $(P=0.673)$.

\section{Discussions}

Bifurcation optimal viewing angles

This study found that both ABOVA and OBOVA distributed sparsely with large ranges of variation in all main coronary bifurcations, indicating that there are no fixed views that can uniformly optimize the visualization of the main coronary bifurcations. The 
Table 2 Bifurcation dimensions assessed by 3D quantitative coronary angiography

\begin{tabular}{|c|c|c|c|c|c|c|}
\hline & \multirow{2}{*}{$\begin{array}{l}\text { ABOVA } \\
\text { Rotation }^{\mathrm{a}}\end{array}$} & \multirow[b]{2}{*}{ Angulation $^{\mathrm{b}}$} & \multicolumn{2}{|l|}{ OBOVA } & \multicolumn{2}{|l|}{ BA } \\
\hline & & & Rotation $^{a}$ & Angulation $^{\mathrm{b}}$ & PBA & DBA \\
\hline LM/LAD/LCx & $5 \pm 33$ & $47 \pm 35$ & $-4 \pm 39$ & $35 \pm 16$ & $128 \pm 24^{\mathrm{c}}$ & $80 \pm 21$ \\
\hline LAD/Diagonal & $4 \pm 38$ & $-50 \pm 14$ & $-14 \pm 28$ & $-33 \pm 5$ & $151 \pm 13$ & $48 \pm 16$ \\
\hline LCx/OM & $-21 \pm 32$ & $27 \pm 17$ & $-18 \pm 31$ & $25 \pm 13$ & $146 \pm 18$ & $57 \pm 16$ \\
\hline PDA/PLA & $-34 \pm 21$ & $-36 \pm 21$ & $-28 \pm 25$ & $-29 \pm 15$ & $145 \pm 19$ & $59 \pm 17$ \\
\hline
\end{tabular}

$A B O V A$ anatomy-defined bifurcation optimal view angle, $O B O V A$ obtainable bifurcation optimal viewing angle, $B A$ bifurcation angle, $P B A$ proximal bifurcation angle, $D B A$ distal bifurcation angle

${ }^{\text {a }}$ Positive value represents right anterior oblique and negative value represents left anterior oblique

b Positive value represents Caudal and negative value represents Cranial

c Angle between LM and LCx

true bifurcation optimal view is subject to the unique anatomy of each individual bifurcation. Given the fact that the viewing angles should be within the reach of the X-ray systems, the optimal view for the left main bifurcation distributes mainly at the Caudal view (35 \pm 16 Caudal) but spreads across the LAO/RAO view ( $439 \mathrm{LAO}$ ); the optimal view for LAD/ Diagonal distributes mainly at the Cranial view (33 \pm 5 Cranial), but spreads across the LAO/RAO view (14 $\pm 28 \mathrm{LAO}$ ); the optimal view for $\mathrm{LCx} / \mathrm{OM}$ distributes mainly at the Caudal view $(25 \pm 13$ Caudal), but spreads across the LAO/RAO view $(18 \pm 31$ LAO); the optimal view for PDA/PLA distributes mainly at the Cranial view $(29 \pm 15$ Cranial) and the LAO view $(28 \pm 25)$.

Three-dimensional quantitative coronary angiography based on routine angiographic projections has emerged as a surrogate to determine optimal viewing angles in the catheterization laboratory. Green [6] evaluated 156 vessel segments and found that vessel foreshortening ranged from 0 to $50 \%$ in the expertrecommended views, while the computer-generated optimal views had only $0.5 \pm 1.2 \%$ foreshortening and less than $2 \%$ overlap. Tu [5] evaluated 67 target vessels by applying a novel overlap prediction approach to avoid overlap in the target vessels and found that the expert viewing angle (EVA) was associated with much more foreshortening as compared with the software viewing angle (SVA), being $8.9 \pm 8.2 \%$ versus. $1.6 \pm 1.5 \% \quad(P<0.001)$. The success of SVA with respect to EVA was also evaluated by two experienced interventional cardiologists and the results were clearly in favor of SVA. Despite the fact that promising results were demonstrated by this new imaging technique, it should be borne in mind that these results were only applied to straight vessels and hence cannot directly be transferred to the more complex bifurcation anatomy [13]. The automated assessment of vessel foreshortening depends on the type of lesion and the number of affected segments. For correct assessment of lesion severity and subsequent precise stent positioning, it might be more critical to minimize overlap between the main vessel and the sidebranch at the ostium.

In a recent study, Tu [3] proposed to use the orthogonal view of the bifurcation as the bifurcation optimal view, since it minimizes the foreshortening and overlap at the ostium. In addition, atherosclerotic plaques occur preferably at the outer lateral wall of the bifurcation, i.e., the site opposite to the carina, where flow is more turbulent and endothelial shear stress is lower. When plaques do involve the carina, they are likely to develop at a later stage of atherosclerosis, as a result of circumferential plaque expansion from the lateral wall [14]. Therefore, the orthogonal viewing angle, i.e., ABOVA, might also expose the lesion severity at its maximum. Nevertheless, ABOVA only minimizes the overlap between the main vessel and the sidebranch at the ostium. Other major coronary arteries could also overlap with the target bifurcation when projected at ABOVA, possibly leading to significant impediment of the visualization of the target bifurcation. To our knowledge, the overlap between the main coronary bifurcations and other coronary arteries at ABOVA has not been documented. Therefore, in this study, we applied the overlap prediction approach to investigate whether this overlap would frequently occur in the general 
population. To our surprise, the overlap of the bifurcation with other major coronary arteries at ABOVA was uncommon. Only three PDA/PLA bifurcations overlapped with the proximal RCA, while the other three main coronary bifurcations had no overlap when projected at ABOVA. This finding further enforces the usefulness of ABOVA. However, due to the mechanical constraints of the current X-ray systems, ABOVA could not be reached in $56.7 \%$ of the population. This occurred more frequent in LM/ LAD/LCx (81.6\%) and LAD/Diagional (78.4\%), followed by PDA/PLA (48.8\%) and was uncommon in LCx/OM (17.6\%). These data suggest that in about half of the population, a second optimal view, i.e., OBOVA, should be used as an alternative by the current X-ray systems. When choosing the second optimal view, priority should be given to minimize the overlap between the main vessel and the sidebranch at the ostium and to maximally expose the lesion severity. In other words, OBOVA for the bifurcation might not be the view elongating the ostial segments at the maximum.

To date, very limited evidence regarding with the use of bifurcation optimal viewing angles in coronary angiography is available in literature. Schlundt [15] demonstrated a case in which sidebranch stenting was performed using the on-line 3D reconstruction of the LAD/Diagonal bifurcation to obtain the optimal view with minimal foreshortening and overlap. Sadamatsu [16] reconstructed 18 left main bifurcations in 3D and selected the optimal views based on foreshortening and overlap. The authors reported that the optimal views were superior to the routine projections in all cases. However, the views were selected to optimize the visualization of the ostial LAD, possibly neglecting the visualization of the LCx. Therefore, these views do not represent the optimal views for the left main bifurcation in the general patient population. To our knowledge, the distributions of ABOVA and OBOVA in main coronary bifurcations have not been previously documented.

\section{Bifurcation angles}

Another finding of this study is that the proximal bifurcation angles (PBAs) as assessed by 3D QCA in LAD/Diagonal, LCx/OM, and PDA/PLA were very much comparable and not statistically different $(P=0.133)$, being $151^{\circ} \pm 13^{\circ}, 146^{\circ} \pm 18^{\circ}$, and $145^{\circ} \pm 19^{\circ}$, respectively. However, the distal bifurcation angles (DBAs) in LAD/Diagonal was smaller than $\mathrm{LCX} / \mathrm{OM} \quad(P=0.004)$ and PDA/PLA $(P=0.001)$, being $48^{\circ} \pm 16^{\circ}$ versus $57^{\circ} \pm 16^{\circ}$, and $59^{\circ} \pm 17^{\circ}$, respectively. The left main bifurcation had the smallest PBA $\left(128^{\circ} \pm 24^{\circ}\right)$ and the largest DBA $\left(80^{\circ} \pm 21^{\circ}\right)$.

Bifurcation angle is an important baseline anatomical characteristic for many randomized bifurcation studies [17]. At present, however, bifurcation angles have been quantified mainly on the basis of $2 \mathrm{D}$ angiographic images, entailing great dependence on the angiographic viewing angle. 3D QCA was proposed to overcome this limitation by measuring the angles in 3D. Tu showed in a bench study that 3D QCA was able to measure bifurcation angles with high accuracy and low variability on a wide range of acquisitions angles [4]. In this study the same 3D QCA software package was used to assess the bifurcation angles in vivo. Our results are very similar to a previous study by Pflederer [18] who evaluated the natural distribution of DBA in 100 patients using multidetector computer tomography $\left(80^{\circ} \pm 27^{\circ}\right.$ in $\mathrm{LM} / \mathrm{LAD} / \mathrm{LCx}, 46^{\circ} \pm 19^{\circ}$ in LAD/Diagonal, $48^{\circ} \pm 24^{\circ}$ in $\mathrm{LCx} / \mathrm{OM}$, and $53^{\circ} \pm 27^{\circ}$ in PDA/PLA). However, another study by Girasis [17] evaluated 266 left main bifurcations using another 3D QCA software package and reported smaller PBA $\left(105.9^{\circ} \pm 21.7^{\circ}\right)$ and larger DBA $\left(95.6^{\circ} \pm 23.6^{\circ}\right)$, as compared with our results. This can be explained by differences in patients and 3D QCA software packages. Since 3D bifurcation reconstruction based on routine angiographic projections needs to correct for various system distortions in vivo [3], different software packages addressing this issue in different approaches might generate discrepancy in the assessed dimensions. In addition, there is no official guideline in the acquisition of angiographic images dedicated for 3D QCA in a broad clinical setting, making the interpretation of different clinical studies difficult.

\section{Limitations}

The study is clearly limited by its retrospective in vivo design. It could therefore not assess whether the applications of bifurcation optimal viewing angles reduced radiation exposure and the volume of contrast medium used, nor improved the diagnosis and outcome of interventional procedures. Further 
prospective studies and randomized clinical trials are warranted before definite conclusions on the added clinical value of software-guided bifurcation optimal viewing angles can be drawn.

\section{Conclusions}

Large variabilities in optimal viewing angles existed for all main coronary bifurcations. The anatomydefined bifurcation optimal viewing angle could not be reached in vivo in roughly half of the cases due to the mechanical constraints of current X-ray systems. Obtainable bifurcation optimal viewing angle should be provided as an alternative or second best. The bifurcation angles in the left main bifurcation demonstrated the largest variabilities.

Conflict of interest Shengxian Tu is employed by Medis medical imaging systems bv and has a research appointment at the Leiden University Medical Center (LUMC). Johan H. C. Reiber is the CEO of Medis medical imaging systems bv, and has a part-time appointment at LUMC as Prof of Medical Imaging.

Open Access This article is distributed under the terms of the Creative Commons Attribution Noncommercial License which permits any noncommercial use, distribution, and reproduction in any medium, provided the original author(s) and source are credited.

\section{References}

1. Iakovou I, Ge L, Colombo A (2005) Contemporary stent treatment of coronary bifurcations. J Am Coll Cardiol 46:1446-1455

2. Suzuki N, Angiolillo DJ, Kawaguchi R, Futamatsu H, Bass TA, Costa MA (2007) Percutaneous coronary intervention of bifurcation coronary disease. Minerva Cardioangiol 55:57-71

3. Lemos PA, Saia F, Ligthart JMR, Arampatzis CA, Sianos G, Tanabe K et al (2003) Coronary restenosis after sirolimuseluting stent implantation: morphological description and mechanistic analysis from a consecutive series of cases. Circulation 108:257-260

4. Mario CD, Sutaria N (2005) Coronary angiography in the angioplasty era: projections with a meaning. Heart 91: 968-976

5. Tu S, Hao P, Koning G, Wei X, Song X, Chen A, Reiber JHC (2011) In vivo assessment of optimal viewing angles from X-ray coronary angiograms. EuroIntervention 7:112-120

6. Green NE, Chen SY, Hansgen AR, Messenger JC, Groves BM, Carroll JD (2005) Angiographic views used for percutaneous coronary interventions: a three-dimensional analysis of physician-determined vs. computer-generated views. Catheter Cardiovasc Interv 64:451-459

7. Tu S, Koning G, Jukema W, Reiber JHC (2010) Assessment of obstruction length and optimal viewing angle from biplane X-ray angiograms. Int $\mathrm{J}$ Cardiovasc Imaging 26:5-17

8. Tu S, Holm N, Koning G, Maeng M, Reiber JHC (2011) The impact of acquisition angle difference on three-dimensional quantitative coronary angiography. Catheter Cardiovasc Interv 78:214-222

9. Tu S, Holm NR, Huang Z, Reiber JHC (2011) Fusion of 3D QCA and IVUS/OCT. Int $\mathrm{J}$ Cardiovasc Imaging 27:197-207

10. Reiber JHC, Serruys PW, Kooijman CJ, Wijns W, Slager CJ, Gerbrands JJ, Schuurbiers JC, den Boer A, Hugenholtz PG (1985) Assessment of short-, medium-, and long-term variations in arterial dimensions from computer-assisted quantitation of coronary cineangiograms. Circulation 71:280-288

11. Tuinenburg JC, Koning G, Rareş A, Janssen JP, Lansky AJ, Reiber JHC (2011) Dedicated bifurcation analysis: basic principles. Int J Cardiovasc Imaging 27:167-174

12. Dumay ACM (1992) Image reconstruction from biplane angiographic projections. Dissertation, Delft University of Technology

13. Lansky A, Tuinenburg J, Costa M, Maeng M, Koning G, Popma J et al (2009) on behalf of the European bifurcation angiographic sub-committee. quantitative angiographic methods for bifurcation lesions: a consensus statement from the European Bifurcation Group. Catheter Cardiovasc Interv 73:258-266

14. Giannoglou GD, Antoniadis AP, Koskinas KC, Chatzizisis YS (2010) Flow and atherosclerosis in coronary bifurcations. EuroIntervention 6(J):16-23

15. Schlundt C, Kreft JG, Fuchs F, Achenbach S, Daniel WD, Ludwig J (2006) Three-dimensional on-line reconstruction of coronary bifurcated lesions to optimize side-branch stenting. Catheter Cardiovasc Interv 68:249-253

16. Sadamatsu K, Sagara S, Yamawaki T, Tashiro H (2009) Three-dimensional coronary imaging for the ostium of the left anterior descending artery. Int $\mathbf{J}$ Cardiovasc Imaging 25:223-228

17. Girasis C, Serruys PW, Onuma Y, Colombo A, Holmes DR Jr, Feldman TE, Bass EJ, Leadley K, Dawkins KD, Morice MC (2010) 3-Dimensional bifurcation angle analysis in patients with left main disease: a substudy of the SYNTAX trial (SYNergy between percutaneous coronary intervention with TAXus and cardiac surgery). JACC Cardiovasc Interv 3:41-48

18. Pflederer T, Ludwig J, Ropers D, Daniel WG, Achenbach S (2006) Measurement of coronary artery bifurcation angles by multidetector computed tomography. Invest Radiol 41:793-798 